\title{
Consensus Analysis of High-Order Multiagent Systems with General Topology and Asymmetric Time-Delays
}

\author{
Fangcui Jiang \\ School of Mathematics and Statistics, Shandong University, Weihai 264209, China \\ Correspondence should be addressed to Fangcui Jiang; jiangfcsdu@sdu.edu.cn
}

Received 4 June 2014; Accepted 22 July 2014; Published 13 August 2014

Academic Editor: Zhichun Yang

Copyright (C) 2014 Fangcui Jiang. This is an open access article distributed under the Creative Commons Attribution License, which permits unrestricted use, distribution, and reproduction in any medium, provided the original work is properly cited.

\begin{abstract}
This paper focuses on the consensus problem for high-order multiagent systems (MAS) with directed network and asymmetric time-varying time-delays. It is proved that the high-order multiagent system can reach consensus when the network topology contains a spanning tree and time-delay is bounded. The main contribution of this paper is that a Lyapunov-like design framework for the explicit selection of protocol parameters is provided. The Lyapunov-like design guarantees the robust consensus of the highorder multiagent system with respect to asymmetric time-delays and is independent of the exact knowledge of the topology when the communication linkages among agents are undirected and connected.
\end{abstract}

\section{Introduction}

In the last few years, substantial research effort from a number of researchers has been poured on the study of consensus problems for multiagent systems (MAS) due to its powerful engineering applications, such as formation control of autonomous vehicles, collective behavior of flocks, and distributed decision making in sensor networks, to name a few. The pioneering contributions in systems and control community have been made by $[1,2]$. Until now, it has been proved that the consensus problem for single-integrator MAS can always be solved under certain mild conditions on the network topology [3-5].

Due to the complexity of real systems, the study on single-integrator MAS can not meet the needs of practical applications. Thus, recently, widespread interest in MAS with agents modeled by general dynamics has been excited among researchers, such as double-integrator model $[6,7]$ and highorder-integrator model. Specifically, high-order-integrator (or high-order) MAS have been studied in [8], where the proposed consensus protocol involves the relative information of all-order derivatives of agents's state. Reference [9] has further extended the partial results of [8] and derived a linearmatrix-inequality-based protocol design. Reference [10] has provided a scheme to choose the coupling strength for fixed and connected topology.

In most practical networks, communication time-delays caused by limited transmission speed and distance cannot be neglected. Within the literature on consensus for MAS with time-delays, recent years have witnessed the introduction of numerous distributed protocols. According to the inducements of time-delays, these protocols can be categorized into the ones in which delays only affect the state information of the agents' neighbors [11], the ones in which delays affect both the agents' own state information and their neighbors' state information $[4,7,12-16]$, the ones containing distributed delays [17], and so forth. In particular, [4] has shown that the consensus of single-integrator MAS might be destabilized by large delays. By using Laplace transform technique, [7] has proved that second-order multiagent systems with bounded and constant time-delays can reach consensus. Based on nonexpansiveness of constant delay operator and Gershgorin's circle theorem, [11] has derived a local controller for high-order MAS with diverse constant time-delays. However, time-varying communication delays are very common in MAS due to the mobility of agents and the disturbance from environment. Hence, it is necessary to study the consensus for 
high-order MAS with time-varying communication delays by exploring other method of protocol design.

In this paper, we investigate the consensus problem for high-order MAS with directed interactions and asymmetric time-varying communication delays. By asymmetric communication delays, we mean that the delay in communication channel from the agent to its neighbor differs from the one in the reversed channel if there are bidirectional communication linkages between them. We assume that the agent can only observe the information of the first state variable of its neighbors, and we propose a distributed protocol by applying both the instantaneous information of the agent's own and the time-delayed relative information with respect to its neighbors. Based on a reduced-order time delay system, we derive some sufficient conditions characterized by linear matrix inequalities (LMIs), according to Lyapunov-Krasovskii functional approach. The main contribution of this paper is that we establish a Lyapunovlike design framework for the explicit selection of protocol parameters. The protocol design framework can not only guarantee the solvability of the LMIs aforementioned but also is independent of the exact knowledge of the topology when the communication linkages among agents are undirected and connected. This implies that the Lyapunov-like protocol design guarantees the robust consensus of high-order MAS with respect to asymmetric time-varying communication delays. Compared with the protocol design in [9], our design is not based on LMI but only needs to solve a simple Lyapunov equation and a simple algebraic inequality. In contrast to the literature on single-/double-integrator MAS, the results here are not simple extensions of the results therein since the protocol parameters have important effect on the consensus convergence of high-order MAS.

The remainder of this paper is organized as follows. Section 2 states the problem formulation and Section 3 presents the main results. Section 4 carries out some numerical examples and the last section provides some concluding remarks.

Notations. We let $\mathbb{R}$ be the set of real numbers. $\mathbb{R}^{n}$ is the $n$-dimensional real vector space. $\mathbb{R}_{n}$ is the set of $n$-by- $n$ matrices with elements in $\mathbb{R}$. $I_{n} \in \mathbb{R}_{n}$ is an identity matrix. Given a matrix $X \in \mathbb{R}_{n}, \sigma(X)$ denotes its spectrum (set of eigenvalues); $X<0$ means that $X$ is negative definite. $\operatorname{diag}\left\{a_{1}, \ldots, a_{n}\right\}$ defines a diagonal matrix with diagonal elements being $a_{1}, \ldots, a_{n}$. Sometimes $0_{m-1} \in \mathbb{R}_{m-1}$ is used to denote zero matrix. Consider $\mathbf{1}_{N}=\left[\begin{array}{lll}1 & \cdots & 1\end{array}\right]^{T} \in \mathbb{R}^{N}$. $\underline{N}=\{1, \ldots, N\}$ and $\underline{m-1}=\{1, \ldots, m-1\}$ are two index sets. $\otimes$ denotes the Kronecker product.

\section{Problem Formulation}

Consider a dynamical system of $N$ autonomous agents, which are labelled 1 through $N$. Each agent is modelled as the following $m$ th order integrator:

$$
\begin{gathered}
\xi_{i}^{(m)}=u_{i}, \quad t \geq 0, i \in \underline{N}, \\
\xi_{i}(0)=\xi_{i 0}, \ldots, \xi_{i}^{(m-1)}(0)=\xi_{i 0}^{(m-1)},
\end{gathered}
$$

where $m \geq 1$ is a positive integer and denotes the order of the differential equations; $\xi_{i} \in \mathbb{R}$, and $\xi_{i}^{(k)}, k=1, \ldots, m$, is the $k$ th order derivative of $\xi_{i} ; u_{i} \in \mathbb{R}$ is the control input; $x_{i}(0):=\left[\begin{array}{llll}\xi_{i 0} & \cdots & \xi_{i 0}^{(m-1)}\end{array}\right]^{T}$ is the initial state of agent $i$.

The interaction/communication topology among agents can be conveniently modeled by weighted directed graph $\mathscr{G}(\mathscr{A})=\{\mathscr{V}, \mathscr{E}, \mathscr{A}\}$, where $\mathscr{V}=\left\{v_{1}, \ldots, v_{N}\right\}$ is the vertex set, $\mathscr{E} \subset \mathscr{V} \times \mathscr{V}$ is the arc set, and $\mathscr{A}=\left[a_{i j}\right] \in \mathbb{R}_{N}$ is the adjacency matrix with $a_{i j} \geq 0$. An arc of $\mathscr{G}$, denoted by $\left(v_{i}, v_{j}\right)$, is an ordered pair of distinct vertices of $\mathscr{V} ; v_{i}$ and $v_{j}$ are called the tail and the head of the arc, respectively. An arc $\left(v_{i}, v_{j}\right) \in \mathscr{E}$ if and only if $a_{j i}>0$. If $\left(v_{i}, v_{j}\right) \in \mathscr{E}$, then we say that $v_{i}$ is a neighbor of $v_{j}$. Denote the collection of neighbors of $v_{i}$ by $\mathcal{N}_{i}=\left\{v_{j}:\left(v_{j}, v_{i}\right) \in \mathscr{E}\right\}$. In this paper, we assume that $\left(v_{i}, v_{i}\right) \notin \mathscr{E}$ and each element of $\mathscr{E}$ is unique. Each vertex in $\mathscr{G}(\mathscr{A})$ represents an agent of the dynamical system (1); $\left(v_{i}, v_{j}\right) \in \mathscr{E}$ indicates that there is a communication linkage from agent $i$ to agent $j$; the element $a_{j i}$ in $\mathscr{A}$ is the weight of the linkage.

A path from $v_{i}$ to $v_{j}$ means that there is a sequence of distinct arcs in $\mathscr{E},\left(v_{i}, v_{1}\right),\left(v_{1}, v_{2}\right), \ldots,\left(v_{k}, v_{j}\right)$. A directed tree is a directed graph, where every vertex has exactly one tail except for one special vertex without any tail. We say a graph contains a spanning tree if there exists a subset of $\operatorname{arcs} \mathscr{E}^{\prime} \subset \mathscr{E}$ such that the graph $\mathscr{G}^{\prime}=\left(\mathscr{V}, \mathscr{E}^{\prime}\right)$ is a directed tree. A graph is said to be balanced if for each vertex $v_{i}$ the weights of its linkages satisfy $\sum_{j=1}^{N} a_{i j}=\sum_{j=1}^{N} a_{j i}, i \in \underline{N}$. A graph is said to be undirected if the associated adjacency matrix $\mathscr{A}$ is symmetric. Then it is easy to see that any undirected graph is balanced. A directed graph is called strongly connected if there exists a path between any two distinct vertices of the graph; for undirected graph it is called connected. An undirected graph is called complete if for any $i \neq j,\left(v_{i}, v_{j}\right) \in \mathscr{E}$. The Laplacian matrix $\mathscr{L}=\left[l_{i j}\right] \in \mathbb{R}_{N}$ of $\mathscr{G}(\mathscr{A})$ is defined as

$$
l_{i j}= \begin{cases}-a_{i j}, & i \neq j \\ \sum_{v_{j} \in \mathscr{N}_{i}} a_{i j}, & i=j .\end{cases}
$$

Let $\mathscr{D}=\operatorname{diag}\left\{d_{1}, \ldots, d_{N}\right\}$ with $d_{i}=\sum_{v_{j} \in \mathcal{N}_{i}} a_{i j}, i \in \underline{N}$. Then $\mathscr{D}$ and $d_{i}$ are called the in-degree matrix of $\mathscr{G}(\mathscr{A})$ and the indegree of vertex $v_{i}$, respectively. From the definition, it is not hard to obtain that $\mathscr{L}=\mathscr{D}-\mathscr{A}$ and $\mathscr{L} \mathbf{1}_{N}=0$. Spectral properties of the Laplacian matrix can be found in $[5,18]$. Hence the details are omitted.

For the system (1), the consensus protocol is described by

$$
\begin{aligned}
u_{i}(t)= & -\sum_{k=1}^{m-1} c_{k} \xi_{i}^{(k)}(t) \\
& -\sum_{j \in \mathcal{N}_{i}} \kappa_{0} a_{i j}\left[\xi_{i}\left(t-\tau_{i j}(t)\right)-\xi_{j}\left(t-\tau_{i j}(t)\right)\right],
\end{aligned}
$$

where $c_{k}>0, k \in \underline{m-1}$ and $\kappa_{0}>0$ are, respectively, the feedback gains of absolute and relative information (for convenience, we refer to the gains $c_{k}, k \in \underline{m-1}$, and $\kappa_{0}$, as the protocol parameters); piecewise continuous function $\tau_{i j}(t)$ is the time-varying delay affecting the communication 
linkage from agent $j$ to agent $i$ at time $t$. Notice that, different from $[4,14,19,20]$, the delays in transmissions from agent $i$ to agent $j$ and from agent $j$ to agent $i$ (if there are bidirectional communication linkages between them) can be asymmetric; that is, $\tau_{i j}(\cdot) \neq \tau_{j i}(\cdot)$.

Remark 1. In protocol (3), the agent $i$ is able to measure its own instantaneous state information and equipped with memories to store the signals $\xi_{i}(\cdot)$ which can be used at some future time and needs only to receive the time-delayed signals $\xi_{j}\left(t-\tau_{i j}(t)\right)$ of its neighbors. The control input $u_{i}(t)$ can be implemented by computing the instantaneous information of all-order derivatives of $\xi_{i}(t)$ and the time-delayed relative information $\xi_{i}\left(t-\tau_{i j}(t)\right)-\xi_{j}\left(t-\tau_{i j}(t)\right)$. The determination of $\tau_{i j}(t)$ can be carried out by assuming that the stored signals $\xi_{i}(\cdot)$ of each agent are time-stamped and neighbor $j$ transmits not only the time-delayed signal $\xi_{j}\left(t-\tau_{i j}(t)\right)$ but also the time stamp. The above situation can be found in $[13,15]$ and also satisfied easily in practice.

Let $x_{i}(t)=\left[\xi_{i}(t) \cdots \xi_{i}^{(m-1)}(t)\right]^{T}$ and $x(0)=$ $\left[x_{1}(0)^{T} \cdots x_{N}(0)^{T}\right]^{T}$ be the state of agent $i$ and the stacked vector of the agents' initial states, respectively. In this paper, we are devoted to solving the following consensus problem for the MAS (1).

Definition 2. Consider the MAS (1) with some given protocol $u_{i}(t)$. If, for any initial state $x(0)$, the states of agents satisfy $x_{i}(t)-x_{j}(t) \rightarrow 0$ as $t \rightarrow \infty$ for all $i, j \in \underline{N}$, then we say that the system solves a consensus problem asymptotically. In addition, if there exists $\xi^{*} \in \mathbb{R}^{m}$ such that, for any initial state $x(0), x_{i}(t) \rightarrow \xi^{*}$ as $t \rightarrow \infty$, for all $i \in \underline{N}$, then we say $\xi^{*}$ is the consensus state of the system.

In order to develop the main results, some helpful lemmas are introduced as follows.

Suppose that $\mathscr{G}_{c}$ is a complete undirected graph of $N$ vertices and $\mathscr{L}_{c}$ is the associated Laplacian matrix. From the definition, we have $\mathscr{L}_{c} \mathbf{1}_{N}=0, \mathbf{1}_{N}^{T} \mathscr{L}_{c}=0$, and the rank of $\mathscr{L}_{c}$ is $N-1$. Let $U_{c}$ be an orthogonal matrix such that $U_{c}^{T} \mathscr{L}_{c} U_{c}=$ $\operatorname{diag}\left\{0, J^{\prime}\right\}$, where $J^{\prime} \in \mathbb{R}_{N-1}$ is a diagonal matrix. Define $U_{c}=\left[\begin{array}{llll}\nu_{1} & v_{2} & \cdots & \nu_{N}\end{array}\right]$ with $\nu_{i} \in \mathbb{R}^{N}, i \in \underline{N}$. It follows that $v_{1}=(1 / \sqrt{N}) \mathbf{1}_{N}$. For convenience, we let $\widehat{\widehat{U}}=\left[v_{2} \cdots v_{N}\right]$. Then $\widehat{U}^{T} \widehat{U}=I_{N-1}$ and $\widehat{U}^{T} \nu_{1}=0$. Based on this observation and the property of Laplacian matrix, we can obtain the result below.

Lemma 3. Suppose that $\mathscr{G}$ is a graph with the associated Laplacian matrix $\mathscr{L}$. Then $U_{c}^{T} \mathscr{L} U_{c}$ is in the form of $\left[\begin{array}{ll}0 & l^{T} \\ 0 & \widehat{L}\end{array}\right]$, where $\widehat{L} \in \mathbb{R}_{N-1}$ and $l \in \mathbb{R}^{N-1} ; \sigma(\widehat{L}) \subset \sigma(\mathscr{L})$. In addition, if $\mathscr{G}$ is balanced, then $l=0$; if $\mathscr{G}$ is strongly connected and balanced, then $\widehat{L}^{T}+\widehat{L}$ is positive definite.

Proof. See the Appendix: Proofs of Lemmas.

The following result can be considered as a special case of Jensen's integral inequality given in [21].
Lemma 4 (see [21]). For any differentiable vector function $y(t) \in \mathbb{R}^{n}$ and any positive definite matrix $P \in \mathbb{R}_{n}$, the following inequality holds:

$$
\begin{gathered}
\tau^{-1}[y(t)-y(t-\tau(t))]^{T} P[y(t)-y(t-\tau(t))] \\
\leq \int_{t-\tau(t)}^{t} \dot{y}^{T}(s) P \dot{y}(s) d s, \quad t \geq 0,
\end{gathered}
$$

where $\tau>0$ and $0 \leq \tau(t) \leq \tau$.

Lemma 5 (Schur complement, see [22]). Let $X, Y, Z$ be some given matrices with appropriate dimensions and let $X, Z$ be symmetric; then $\left[\begin{array}{cc}X & Y \\ Y^{T} & Z\end{array}\right]<0$ if and only if $X<0, Z-Y^{T} X^{-1} Y<$ 0 , or $Z<0, X-Y Z^{-1} Y^{T}<0$.

\section{Main Results}

In this section, we first provide an equivalent condition for the consensus convergence of the systems (1) and (3) based on an orthogonal state transformation and a reduced-order time delay system. Then we give a Lyapunov-like parameter design for the protocol and prove that the maximum allowable upper bounds of time-varying delays can be determined by solving some optimization problems.

Suppose that the interaction topology of the system (1) is modelled by $\mathscr{G}(\mathscr{A})$; the associated Laplacian matrix is $\mathscr{L}$. Then the dynamics of agent $i$ can be written as

$$
\begin{aligned}
\dot{x}_{i}(t)= & E_{m} x_{i}(t)-\sum_{j \in \mathcal{N}_{i}} \kappa_{0} a_{i j} \\
& \times F_{m}\left[x_{i}\left(t-\tau_{i j}(t)\right)-x_{j}\left(t-\tau_{i j}(t)\right)\right],
\end{aligned}
$$

where

$$
\begin{gathered}
E_{m}=\left[\begin{array}{cc}
0 & I_{m-1} \\
0 & \theta^{T}
\end{array}\right], \quad F_{m}=\left[\begin{array}{cc}
0 & 0 \\
1 & 0
\end{array}\right], \\
\theta=\left[\begin{array}{llll}
-c_{1} & -c_{2} & \cdots & -c_{m-1}
\end{array}\right]^{T} .
\end{gathered}
$$

Denote $x(t)=\left[\begin{array}{llll}x_{1}^{T}(t) & \cdots & x_{N}^{T}(t)\end{array}\right]^{T}$ by the stacked vector of the agents' states. The closed-loop dynamics of the systems (1) and (3) are in the form of

$$
\dot{x}(t)=\left(I_{N} \otimes E_{m}\right) x(t)-\sum_{k=1}^{M}\left(\kappa_{0} \mathscr{L}_{k} \otimes F_{m}\right) x\left(t-\tau_{k}(t)\right),
$$

where $M$ denotes the number of different time-delays over the communication channels of the system (it is easy to get that $M \leq N(N-1)) ; \tau_{k}(t) \in\left\{\tau_{i j}(t): i, j \in \underline{N}, i \neq j\right\}$ for $k=1, \ldots, M ; \mathscr{L}_{k}=\left[l_{i j}^{k}\right] \in \mathbb{R}_{N}$ is defined as

$$
l_{i j}^{k}= \begin{cases}-a_{i j}, & j \neq i, \quad \tau_{i j}(\cdot)=\tau_{k}(\cdot), \\ 0, & j \neq i, \quad \tau_{i j}(\cdot) \neq \tau_{k}(\cdot), \\ -\sum_{n=1}^{N} l_{i n}^{k}, & j=i .\end{cases}
$$


Next, we assume that the time-delays in (7) satisfy

$$
0 \leq \tau_{k}(t) \leq \tau_{k}, \quad t \geq 0, k=1, \ldots, M
$$

where $\tau_{k}>0$. Define $\tau_{0}=\max \left\{\tau_{k}: k=1, \ldots, M\right\}$. The initial state of the system (7) is assumed to be $\phi(t)=x(0)$, $t \in\left[-\tau_{0}, 0\right]$.

Remark 6. The matrix $\mathscr{L}_{k}$ in (7) amounts to consider a Laplacian matrix that is associated with a subgraph of $\mathscr{G}(\mathscr{A})$ with all linkages affected by the same time-delay. Hence, $\mathscr{L}_{k} \mathbf{1}_{N}=0$ and $\sum_{k=1}^{M} \mathscr{L}_{k}=\mathscr{L}$. According to Lemma 3 , we can let $U_{c}^{T} \mathscr{L}_{k} U_{c}=\left[\begin{array}{cc}0 & l_{k}^{T} \\ 0 & \widehat{L}_{k}\end{array}\right], U_{c}^{T} \mathscr{L} U_{c}=\left[\begin{array}{cc}0 & l^{T} \\ 0 & \widehat{L}\end{array}\right]$, where $U_{c}$ is given as therein. Then $\sum_{k=1}^{M} \widehat{L}_{k}=\widehat{L}$. Furthermore, it is important to point out that even when the topology $\mathscr{G}(\mathscr{A})$ is undirected, $\mathscr{L}_{k}$ is unnecessarily symmetric due to $\tau_{i j}(\cdot) \neq$ $\tau_{j i}(\cdot)$. Consequently, the results of $[4,14,19,20]$ are invalid to deal with the consensus problem for MAS with asymmetric communication delays.

Applying the orthogonal linear transformation $x(t)=$ $\left(U_{c} \otimes I_{m}\right) y(t)$ to the system (7) and denoting $y(t)=$ $\left[\begin{array}{ll}y_{1}^{T}(t) & y_{2}^{T}(t)\end{array}\right]^{T}$ with $y_{1}(t) \in \mathbb{R}^{m}$ and $y_{2}(t) \in \mathbb{R}^{(N-1) m}$, we have

$$
\begin{gathered}
\dot{y}_{1}(t)=E_{m} y_{1}(t)-\sum_{k=1}^{M}\left(\kappa_{0} l_{k}^{T} \otimes F_{m}\right) y_{2}\left(t-\tau_{k}(t)\right), \\
\dot{y}_{2}(t)=\left(I_{N-1} \otimes E_{m}\right) y_{2}(t)-\sum_{k=1}^{M}\left(\kappa_{0} \widehat{L}_{k} \otimes F_{m}\right) y_{2}\left(t-\tau_{k}(t)\right),
\end{gathered}
$$

where $l_{k}$ and $\widehat{L}_{k}$ are defined as in Remark 6. It can be seen that the system (11) is independent of the dynamics of $y_{1}(t)$ and has the order $(N-1) m$; the system (10) can be regarded as a forced system with the forced motion caused by the delayed state of $y_{2}(t)$. From $U_{c}=\left[(1 / \sqrt{N}) \mathbf{1}_{N} \widehat{U}\right]$ and some direct computation, it follows that

$$
x(t)=\left(U_{c} \otimes I_{m}\right) y(t)=\frac{1}{\sqrt{N}} \mathbf{1}_{N} \otimes y_{1}(t)+\left(\widehat{U} \otimes I_{m}\right) y_{2}(t) .
$$

In addition, Proposition 7 will indicate that $y_{2}(t)$ can be considered as the disagreement state of the system (7). Note that, for the time delay system in (10) and (11), we assume that the initial state is $\varphi(t)=\left(U_{c}^{T} \otimes I_{m}\right) x(0) \triangleq\left[\begin{array}{ll}y_{10}^{T} & y_{20}^{T}\end{array}\right]^{T}$, $t \in\left[-\tau_{0}, 0\right]$, where $y_{10} \in \mathbb{R}^{m}, y_{20} \in \mathbb{R}^{(N-1) m}$, and $\tau_{0}$ is given in (7).

We are now in a position to present an equivalent condition for the consensus convergence of the system (7).

Proposition 7. Consider the system (7) with topology $\mathscr{G}(\mathscr{A})$. The system solves a consensus problem asymptotically if and only if each solution of the reduced-order system (11) converges to zero.

Proof. Necessity. Suppose that $y_{2}(t)$ is any solution of the system (11) with initial state $\varphi_{2}(t)=y_{20} \in \mathbb{R}^{(N-1) m}$, $t \in\left[-\tau_{0}, 0\right]$. Let $y_{1}(t) \in \mathbb{R}^{m}$ be continuously differentiable and satisfy the dynamics (10) with initial state $y_{10}$. From (12), it follows that $x(t)=(1 / \sqrt{N}) \mathbf{1}_{N} \otimes y_{1}(t)+\left(\widehat{U} \otimes I_{m}\right) y_{2}(t)$ is a solution of the system (7) with initial state $\phi(t)=$ $\left(U_{c} \otimes I_{m}\right)\left[\begin{array}{ll}y_{10}^{T} & y_{20}^{T}\end{array}\right]^{T}, t \in\left[-\tau_{0}, 0\right]$. Denote $\left(\widehat{U} \otimes I_{m}\right) y_{2}(t)=$ $\left[z_{1}^{T}(t) \cdots z_{N}^{T}(t)\right]^{T}$ with $z_{i}(t) \in \mathbb{R}^{m}, i \in \underline{N}$. Then (12) implies that $z_{j}(t)-z_{1}(t)=x_{j}(t)-x_{1}(t), j=2, \ldots, N$, where $x_{j}(t)$ is the state of agent $j$. If the system (7) solves a consensus problem asymptotically, then $x_{j}(t)-x_{1}(t) \rightarrow 0$ as $t \rightarrow \infty, j=$ $2, \ldots, N$. Hence $z_{j}(t)-z_{1}(t) \rightarrow 0$ as $t \rightarrow \infty, j=2, \ldots, N$, which is equivalent to $\left(\widehat{U} \otimes I_{m}\right) y_{2}(t)=\left[\begin{array}{llll}z_{1}^{T}(t) & \cdots & z_{N}^{T}(t)\end{array}\right]^{T} \rightarrow$ $\mathbf{1}_{N} \otimes z_{1}(t)$. Due to $\widehat{U}^{T} \widehat{U}=I_{N-1}$ and $\widehat{U}^{T} \mathbf{1}_{N}=0, y_{2}(t)=$ $\left(\widehat{U}^{T} \otimes I_{m}\right)\left(\widehat{U} \otimes I_{m}\right) y_{2}(t) \rightarrow\left(\widehat{U}^{T} \otimes I_{m}\right)\left(\mathbf{1}_{N} \otimes z_{1}(t)\right)=0$. Hence each solution of the system (11) converges to zero.

Sufficiency. Suppose that each solution of the system (11) converges to zero, and $x(t)$ is the solution of the system (7) with any initial state $\varphi(t)=x(0), t \in\left[-\tau_{0}, 0\right]$. Let $y(t)=\left(U_{c}^{T} \otimes I_{m}\right) x(t) \triangleq\left[y_{1}^{T}(t) y_{2}^{T}(t)\right]^{T}$, where $y_{1}(t) \in \mathbb{R}^{m}$, $y_{2}(t) \in \mathbb{R}^{(N-1) m}$. Then $y(t)$ is a solution of the systems (10) and (11) with initial state $\varphi(t)=\left(U_{c}^{T} \otimes I_{m}\right) x(0), t \in\left[-\tau_{0}, 0\right]$. The assumption implies that $y_{2}(t) \rightarrow 0$ as $t \rightarrow \infty$. Due to $(12), \lim _{t \rightarrow \infty} x_{i}(t)=(1 / \sqrt{N}) \lim _{t \rightarrow \infty} y_{1}(t), i \in \underline{N}$ in which $y_{1}(t)$ evolves according to (10). This indicates that the system (7) solves a consensus problem asymptotically with the consensus state $(1 / \sqrt{N}) \lim _{t \rightarrow \infty} y_{1}(t)$.

Remark 8. It should be pointed out that the orthogonal linear transformation $x(t)=\left(U_{c} \otimes I_{m}\right) y(t)$ is not uniquely defined by $U_{c}$. Actually, any orthogonal matrix with the first column being $(1 / \sqrt{N}) \mathbf{1}_{N}$ also can derive the result of Proposition 7 . In addition, the above orthogonal linear transformation can be seen as an improvement of the transformation on disagreement space which was displayed in Lemma 5.2 of [20].

Remark 9. In contrast to the transformation (7) of [15] and the tree-type transformation of [16], the order of the reduced-order system (11) induced by the orthogonal linear transformation $x(t)=\left(U_{c} \otimes I_{m}\right) y(t)$ is lower than those of the reduced-order systems induced by them (in the context that all of the three transformations are applied to the system (7)). It is easy to see that the order of the reduced-order system (11) is $N m-m$, whereas the order of the reducedorder system which is derived from the transformation (7) of [15] (or the tree-type transformation of [16]) is $N m-$ 1. Seen from the linear-matrix-inequality-based sufficient conditions which will be given in Theorem 12, our orthogonal linear transformation can derive lower-order linear matrix inequalities. This will reduce the computation cost to some extent when estimating the maximum allowable upper bound $\tau_{0}$ of time-varying delays.

Before presenting the main result, the following lemmas are introduced to give the parameter design of protocol (3). 
Lemma 10. Consider $E_{m}$ and $F_{m}$ in (5). Suppose that $E_{m}$ has only one zero eigenvalue and other nonzero eigenvalues have negative real parts, and $\kappa_{0}>0$ is a constant. Let $\widehat{Q}_{1}=$ $\operatorname{diag}\left\{0, q_{2}, \ldots, q_{m}\right\}$ with $q_{i}>0, i=2, \ldots, m ; P_{m}=\left[p_{i j}\right] \in$ $\mathbb{R}_{m}$ is a positive definite solution of the Lyapunov equation $E_{m}^{T} P_{m}+P_{m} E_{m}=-\widehat{Q}_{1} ; z=\alpha+\sqrt{-1} \beta$ is a complex number with $\alpha>0 ; D_{z}=\left[\begin{array}{cc}\alpha & \beta \\ -\beta & \alpha\end{array}\right]$. Then $\left(I_{2} \otimes E_{m}-\kappa_{0} D_{z} \otimes F_{m}\right)^{T}\left(I_{2} \otimes\right.$ $\left.P_{m}\right)+\left(I_{2} \otimes P_{m}\right)\left(I_{2} \otimes E_{m}-\kappa_{0} D_{z} \otimes F_{m}\right)<0$ if and only if

$$
\kappa_{0}<\frac{2 \alpha p_{1 m}}{\left(\alpha^{2}+\beta^{2}\right) \sum_{i=2}^{m}\left(p_{i m}^{2} / q_{i}\right)} .
$$

In particular, when $\beta=0,\left(E_{m}-\kappa_{0} \alpha F_{m}\right)^{T} P_{m}+P_{m}\left(E_{m}-\right.$ $\left.\kappa_{0} \alpha F_{m}\right)<0$ if and only if $\kappa_{0}<2 p_{1 m} / \alpha \sum_{i=2}^{m}\left(p_{i m}^{2} / q_{i}\right)$.

Proof. See the Appendix: Proofs of Lemmas.

Lemma 11. Suppose that $\mathscr{G}(\mathscr{A})$ contains a spanning tree; the associated Laplacian matrix is $\mathscr{L}, U_{c}^{T} \mathscr{L} U_{c}=\left[\begin{array}{cc}0 & T^{T} \\ 0 & \widehat{L}\end{array}\right]$; the parameters $c_{i}, i \in \underline{m-1}$ are chosen such that $E_{m}$ has only one zero eigenvalue and other nonzero eigenvalues have negative real parts; $\widehat{Q}_{1}=\operatorname{diag}\left\{0, q_{2}, \ldots, q_{m}\right\}$ with $q_{i}>0, i=2, \ldots, m$, and $P_{m}=\left[p_{i j}\right] \in \mathbb{R}_{m}$ is a positive definite solution of the Lyapunov equation $E_{m}^{T} P_{m}+P_{m} E_{m}=-\widehat{Q}_{1}$. If

$$
\begin{gathered}
0<\kappa_{0}<\min \left\{\frac{2 r p_{1 m}}{\left(r^{2}+d^{2}\right) \sum_{i=2}^{m}\left(p_{i m}^{2} / q_{i}\right)},\right. \\
\left.\frac{4 p_{1 m}}{5 d \sum_{i=2}^{m}\left(p_{i m}^{2} / q_{i}\right)}\right\},
\end{gathered}
$$

then $I_{N-1} \otimes E_{m}-\kappa_{0} \widehat{L} \otimes F_{m}$ is Huiwitz stable, where $r=$ $\min \{\operatorname{Re}(\lambda): \lambda \in \sigma(\mathscr{L}) \backslash\{0\}\}$ and $d=\max _{i \in N}\left\{d_{i}\right\}$ is the maximum vertex in-degree over $\mathscr{G}(\mathscr{A})$.

Proof. See the Appendix: Proofs of Lemmas.

By choosing the parameters $c_{i}, i \in m-1$ and $\kappa_{0}$ as in Lemma 11 and making use of Proposition 7 , we can obtain the following theorem.

Theorem 12. Consider the system (7) with fixed topology $\mathscr{G}(\mathscr{A})$. Suppose that $\mathscr{G}(\mathscr{A})$ contains a spanning tree; the associated Laplacian matrix $\mathscr{L}$ has the form of $U_{c}^{T} \mathscr{L} U_{c}=$ $\left[\begin{array}{ll}0 & l^{T} \\ 0 & \widehat{L}\end{array}\right]$, where $U_{c}$ is given as in Lemma 3; the parameters $c_{i}$, $i \in \underline{m-1}$ and $\kappa_{0}$ are chosen as in Lemma 11. Then the system solves a consensus problem asymptotically if the time-varying delays satisfy $\tau_{i j}(t) \leq \tau_{0}$, where $\tau_{0}$ is obtained from the following optimization problem:

$$
\begin{aligned}
\text { maximize } & \tau_{0} \\
\text { subject to } & {\left[\begin{array}{ccc}
G^{T} P+P G & P H & \Phi_{13} \\
* & 0 & \Phi_{23} \\
* & * & 0
\end{array}\right] } \\
& <\tau_{0}^{-1} \operatorname{diag}\left\{0, \Phi_{0}, \Phi_{0}\right\}, \\
& P>0, \quad Q_{k}>0, \quad k=1, \ldots, M,
\end{aligned}
$$

where

$$
\begin{aligned}
G & =I_{N-1} \otimes E_{m}-\kappa_{0} \widehat{L} \otimes F_{m}, \\
H & =\left[\kappa_{0} \widehat{L}_{1} \otimes F_{m} \cdots \kappa_{0} \widehat{L}_{M} \otimes F_{m}\right], \\
\Phi_{13} & =\left[G^{T} Q_{1} \cdots G^{T} Q_{M}\right], \\
\Phi_{23} & =\left[H^{T} Q_{1} \cdots H^{T} Q_{M}\right], \\
\Phi_{0} & =\operatorname{diag}\left\{Q_{1}, \ldots, Q_{M}\right\},
\end{aligned}
$$

and “*" represents the elements below the main diagonal of a symmetric matrix.

Proof. We first show that there must exist some positive definite matrices $P$ and $Q_{k}$ such that the first LMI in (15) is solvable. From the selection of protocol parameters and Lemma 11, we know that $G$ is Hurwitz stable. Hence there is a positive definite matrix $\widetilde{P}$ such that $G^{T} \widetilde{P}+\widetilde{P} G<0$. By Lemma 5, the first LMI in (15) is equivalent to

$$
\begin{aligned}
& \underbrace{\left[\begin{array}{cc}
G^{T} P+P G & P H \\
* & -\tau_{0}^{-1} \Phi_{0}
\end{array}\right]}_{\triangleq \Sigma_{1}}+\left(\mathbf{1}_{M}^{T} \otimes\left[\begin{array}{c}
G^{T} \\
H^{T}
\end{array}\right]\right) \tau_{0} \Phi_{0} \\
& \quad \times\left(\mathbf{1}_{M} \otimes\left[\begin{array}{ll}
G & H
\end{array}\right]\right)<0,
\end{aligned}
$$

and $\Sigma_{1}<0$ is equivalent to $G^{T} P+P G+\tau_{0} P H \Phi_{0}^{-1} H^{T} P<0$. Consequently, if we choose $P=\widetilde{P}, Q_{k}=I_{(N-1) m}, k=$ $1, \ldots, M$, then for some sufficiently small $\tau_{0}, G^{T} \widetilde{P}+\widetilde{P} G+$ $\tau_{0} \widetilde{P} H H^{T} \widetilde{P}<0$ holds. This means that $P=\widetilde{P}$ and $Q_{k}=$ $I_{(N-1) m}$ satisfy the LMIs in (15).

According to Proposition 7, it suffices to prove that the zero solution of the reduced-order system (11) is asymptotically stable under the condition (15). To do this, consider the following Lyapunov-Krasovskii functional candidate:

$$
\begin{aligned}
V\left(y_{2}(t)\right)= & y_{2}^{T}(t) P y_{2}(t) \\
& +\sum_{k=1}^{M} \int_{-\tau_{k}}^{0} \int_{t+\theta}^{t} \dot{y}_{2}^{T}(s) Q_{k} \dot{y}_{2}(s) d s d \theta .
\end{aligned}
$$


Note that Remark 6 implies that $\sum_{k=1}^{M} \widehat{L}_{k}=\widehat{L}$. Then the time derivative of $V\left(y_{2}(t)\right)$ along the trajectory of (11) is

$$
\begin{aligned}
\dot{V}\left(y_{2}(t)\right)= & 2 y_{2}^{T}(t) P \dot{y}_{2}(t) \\
& +\sum_{k=1}^{M}\left[\tau_{k} \dot{y}_{2}^{T}(t) Q_{k} \dot{y}_{2}^{T}(t)\right. \\
& \left.-\int_{-\tau_{k}}^{0} \dot{y}_{2}^{T}(t+\theta) Q_{k} \dot{y}_{2}(t+\theta) d \theta\right] \\
= & 2 y_{2}^{T}(t) P G y_{2}(t) \\
& +2 y_{2}^{T}(t) P\left[\sum_{k=1}^{M} \kappa_{0}\left(\widehat{L}_{k} \otimes F_{m}\right)\right. \\
& +\sum_{k=1}^{M}\left[\tau_{k} \dot{y}_{2}^{T}(t) Q_{k} \dot{y}_{2}(t)\right. \\
& \left.\quad \int_{-\tau_{k}}^{0} \dot{y}_{2}^{T}(t+\theta) Q_{k} \dot{y}_{2}(t+\theta) d \theta\right]
\end{aligned}
$$$$
\leq 2 y_{2}^{T}(t) P G y_{2}(t)
$$$$
+2 y_{2}^{T}(t) P\left[\sum_{k=1}^{M} \kappa_{0}\left(\widehat{L}_{k} \otimes F_{m}\right)\right.
$$$$
\left.\times\left(y_{2}(t)-y_{2}\left(t-\tau_{k}(t)\right)\right)\right]
$$$$
+\sum_{k=1}^{M}\left[\tau_{k} \dot{y}_{2}^{T}(t) Q_{k} \dot{y}_{2}(t)\right.
$$$$
\left.-\int_{t-\tau_{k}(t)}^{t} \dot{y}_{2}^{T}(s) Q_{k} \dot{y}_{2}(s) d s\right]
$$$$
\leq 2 y_{2}^{T}(t) P G y_{2}(t)
$$$$
+2 y_{2}^{T}(t) P\left[\sum_{k=1}^{M} \kappa_{0}\left(\widehat{L}_{k} \otimes F_{m}\right)\right.
$$$$
\left.\times\left(y_{2}(t)-y_{2}\left(t-\tau_{k}(t)\right)\right)\right]
$$

$$
\begin{aligned}
+\sum_{k=1}^{M}\left\{\tau_{k} \dot{y}_{2}^{T}(t) Q_{k} \dot{y}_{2}(t)\right. \\
-\tau_{k}^{-1}\left[y_{2}(t)-y_{2}\left(t-\tau_{k}(t)\right)\right]^{T} \\
\left.\quad \times Q_{k}\left[y_{2}(t)-y_{2}\left(t-\tau_{k}(t)\right)\right]\right\},
\end{aligned}
$$

where $G$ is given as in (15). Let $z_{k}(t)=y_{2}(t)-y_{2}\left(t-\tau_{k}(t)\right)$ and replace $\dot{y}_{2}(t)$ with the right-hand term of (11). It follows that

$$
\begin{aligned}
& \dot{V}\left(y_{2}(t)\right) \leq 2 y_{2}^{T}(t) P G y_{2}(t) \\
& +2 y_{2}^{T}(t) P\left[\sum_{k=1}^{M} \kappa_{0}\left(\widehat{L}_{k} \otimes F_{m}\right) z_{k}(t)\right] \\
& -\sum_{k=1}^{M} \tau_{0}^{-1} z_{k}^{T}(t) Q_{k} z_{k}(t) \\
& +\sum_{k=1}^{M}\left\{\tau _ { 0 } \left[G y_{2}(t)\right.\right. \\
& \left.+\sum_{k=1}^{M} \kappa_{0}\left(\widehat{L}_{k} \otimes F_{m}\right) z_{k}(t)\right]^{T} \\
& \times Q_{k}\left[G y_{2}(t)\right. \\
& \left.\left.+\sum_{k=1}^{M} \kappa_{0}\left(\widehat{L}_{k} \otimes F_{m}\right) z_{k}(t)\right]\right\} \\
& =\left[y_{2}^{T}(t) Z^{T}(t)\right] \\
& \times\left\{\left[\begin{array}{cc}
G^{T} P+P G & P H \\
* & -\tau_{0}^{-1} \Phi_{0}
\end{array}\right]\right. \\
& \left.+\left[\begin{array}{ccc}
G^{T} & \cdots & G^{T} \\
H^{T} & \cdots & H^{T}
\end{array}\right] \tau_{0} \Phi_{0}\left[\begin{array}{cc}
G & H \\
\vdots & \vdots \\
G & H
\end{array}\right]\right\}\left[\begin{array}{c}
y_{2}(t) \\
Z(t)
\end{array}\right] \\
& =\left[y_{2}^{T}(t) Z^{T}(t)\right] \\
& \times\left\{\left[\begin{array}{cc}
G^{T} P+P G & P H \\
* & -\tau_{0}^{-1} \Phi_{0}
\end{array}\right]\right. \\
& \left.-\left[\begin{array}{c}
\Phi_{13} \\
\Phi_{23}
\end{array}\right]\left(-\tau_{0}^{-1} \Phi_{0}\right)^{-1}\left[\Phi_{13}^{T} \Phi_{23}^{T}\right]\right\}\left[\begin{array}{c}
y_{2}(t) \\
Z(t)
\end{array}\right],
\end{aligned}
$$

where $Z(t)=\left[z_{1}^{T}(t) \cdots z_{M}^{T}(t)\right]^{T} ; H, \Phi_{0}, \Phi_{13}$, and $\Phi_{23}$ are given as in (15). From Lemma 5 and (15), we have $\dot{V}\left(y_{2}(t)\right)<$ 0 . Hence there exists a positive real number $a_{0}$ such that

$$
\begin{aligned}
\dot{V}\left(y_{2}(t)\right) & <-a_{0}\left[y_{2}^{T}(t) y_{2}(t)+Z^{T}(t) Z(t)\right] \\
& <-a_{0} y_{2}^{T}(t) y_{2}(t) .
\end{aligned}
$$

This proves that the zero solution of the reduced-order system (11) is asymptotically stable. Thus the conclusion holds. 
Remark 13. From the proof of Theorem 12, it can be seen that the parameter design of protocol (3) in the theorem (i.e., the selection of $c_{i}, i \in \underline{m-1}$, and $\kappa_{0}$ ) guarantees the solvability of the LMIs in $(15)$. According to the nature of the parameter design (see Lemma 11), we refer to it as a Lyapunov-like parameter design.

In Lemma 11, when $\mathscr{G}(\mathscr{A})$ is undirected and connected, $\widehat{L}$ is positive definite. Hence $\lambda_{i} \in \sigma(\mathscr{L}) \backslash\{0\}, i=$ $2, \ldots, N$, are positive real numbers, $r=\min \left\{\lambda_{2}, \ldots, \lambda_{N}\right\}$ and $r \leq \lambda_{i} \leq 2 d$. In this case, for any $\kappa_{0}: 0<$ $\kappa_{0}<\min \left\{2 p_{1 m} / r \sum_{i=2}^{m}\left(p_{i m}^{2} / q_{i}\right), p_{1 m} / d \sum_{i=2}^{m}\left(p_{i m}^{2} / q_{i}\right)\right\}=$ $p_{1 m} / d \sum_{i=2}^{m}\left(p_{i m}^{2} / q_{i}\right), G=I_{N-1} \otimes E_{m}-\kappa_{0} \widehat{L} \otimes F_{m}$ is Hurwitz stable. From Lemma 10, it follows that when $0<\kappa_{0}<$ $p_{1 m} / d \sum_{i=2}^{m}\left(p_{i m}^{2} / q_{i}\right),\left(E_{m}-\kappa_{0} \lambda_{i} F_{m}\right)^{T} P_{m}+P_{m}\left(E_{m}-\kappa_{0} \lambda_{i} F_{m}\right)<0$ for any $\lambda_{i} \in \sigma(\mathscr{L}) \backslash\{0\}$. Since $\widehat{L}$ is positive definite, there is an orthogonal matrix $S$ such that $S^{T} \widehat{L} S=\operatorname{diag}\left\{\lambda_{2}, \ldots, \lambda_{N}\right\}$. Thus when $0<\kappa_{0}<p_{1 m} / d \sum_{i=2}^{m}\left(p_{i m}^{2} / q_{i}\right), G^{T}\left(I_{N-1} \otimes P_{m}\right)+$ $\left(I_{N-1} \otimes P_{m}\right) G=\left(S \otimes I_{m}\right)\left[\left(I_{N-1} \otimes E_{m}-\kappa_{0} \operatorname{diag}\left\{\lambda_{2}, \ldots, \lambda_{N}\right\} \otimes\right.\right.$ $\left.F_{m}\right)^{T}\left(I_{N-1} \otimes P_{m}\right)+\left(I_{N-1} \otimes P_{m}\right)\left(I_{N-1} \otimes E_{m}-\kappa_{0} \operatorname{diag}\left\{\lambda_{2}, \ldots, \lambda_{N}\right\} \otimes\right.$ $\left.\left.F_{m}\right)\right]\left(S^{T} \otimes I_{m}\right)<0$. In the light of the proof of Theorem 12, the inequalities in (15) hold by taking $P=I_{N-1} \otimes P_{m}, Q_{k}=I_{(N-1) m}$, and sufficiently small $\tau_{0}$. This result can be summarized as the following corollary.

Corollary 14. Consider the system (7) with fixed topology $\mathscr{G}(\mathscr{A})$. Suppose that $\mathscr{G}(\mathscr{A})$ is undirected and connected; the parameters $c_{i}, i \in \underline{m-1}$, are chosen such that $E_{m}$ has only one zero eigenvalue and other nonzero eigenvalues have negative real parts; $\widehat{Q}_{1}=\operatorname{diag}\left\{0, q_{2}, \ldots, q_{m}\right\}$ with $q_{i}>0, i=2, \ldots, m$, and $P_{m}=\left[p_{i j}\right] \in \mathbb{R}_{m}$ is a positive definite solution of the Lyapunov equation $E_{m}^{T} P_{m}+P_{m} E_{m}=-\widehat{Q}_{1} ; \kappa_{0}$ satisfies

$$
0<\kappa_{0}<\frac{p_{1 m}}{d \sum_{i=2}^{m}\left(p_{i m}^{2} / q_{i}\right)},
$$

where $d=\max _{i \in N}\left\{d_{i}\right\}$ is the maximum vertex in-degree over $\mathscr{G}(\mathscr{A})$. Then the system solves a consensus problem asymptotically if the time-varying delays satisfy $\tau_{i j}(t) \leq \tau_{0}$, where $\tau_{0}$ is obtained from (15) with $P=I_{N-1} \otimes P_{m}$ and $Q_{k}=I_{(N-1) m}$.

Remark 15. Corollary 14 indicates that the selection of parameters $c_{i}, i \in \underline{m-1}$, and $\kappa_{0}$ is independent of the eigenvalues of Laplacian matrix but only depends on the maximum vertex in-degree of the graph. It will reduce greatly the computation and storage costs for the protocol design of practical MAS. Therefore, we can say that the parameter design in Theorem 12 is independent of the precise interaction topology when the underlying graph is connected and guarantees the robust consensus with respect to asymmetric time-varying delays for the high-order MAS (7).

Remark 16. It is worth pointing out that the results of [19] can not be applied to the high-order MAS straightforwardly, since the parameters $c_{j}, j \in \underline{m-1}$, and $\kappa_{0}$ have important effect on the consensus of the system. Whereas the Lyapunovlike parameter design given in Theorem 12 can solve the consensus problem for the high-order MAS (7) very well.
Remark 17. Compared with the existing results, the main contribution of this paper is giving the Lyapunov-like parameter design which is easy to implement, independent of the precise interaction topology for the case of connected graphs, and robust with respect to asymmetric time-varying delays. Moreover, the parameter design can guarantee the existence of solution of the linear matrix inequalities given in Theorem 12, although it seems that the estimations of $\tau_{0}$ are conservative. More excellent estimation on the maximum allowable upper bound of time-varying delays is a commonly unsolved problem. This requires us to explore other analysis techniques which could reduce the dependence of that estimation on the knowledge of network topology.

\section{Numerical Examples}

Consider the system (7) of six agents with dynamics described by a triple-order integrator. The interaction topology among agents is depicted by a cycle with the $\operatorname{arcs}\left(v_{1}, v_{2}\right)$, $\left(v_{2}, v_{3}\right),\left(v_{3}, v_{4}\right),\left(v_{4}, v_{5}\right),\left(v_{5}, v_{6}\right),\left(v_{6}, v_{1}\right)$. Assume that the weights of the arcs are $a_{21}=a_{32}=a_{43}=a_{54}=a_{65}=a_{16}=$ 1 and the communication delays affecting on the linkages are different from each other. Then the maximum vertex indegree of the graph is $d=1$, and the minimum real part of nonzero eigenvalues of the associated Laplacian matrix is $r=1 / 2$.

In what follows, we design the protocol parameters $c_{1}, c_{2}$, and $\kappa_{0}$ according to Theorem 12 (or Lemma 11).

It is not hard to obtain that the characteristic polynomial of $E_{3}$ in the system (7) is $s\left(s^{2}+c_{2} s+c_{1}\right)$. Then any positive numbers $c_{1}$ and $c_{2}$ make $E_{3}$ satisfy the assumption in Lemma 11. Let $P_{3}=\left[p_{i j}\right] \in \mathbb{R}_{3}$ and $\widehat{Q}_{1}=\operatorname{diag}\left\{0, q_{2}, q_{3}\right\}$ with $q_{2}>0, q_{3}>0$. By solving $E_{3}^{T} P_{3}+P_{3} E_{3}=-\widehat{Q}_{1}$, we have $p_{11}=c_{1} p_{13}, p_{12}=c_{2} p_{13}, p_{22}=\left(c_{2}^{2} / c_{1}\right) p_{13}+$ $\left(c_{2} / 2 c_{1}+1 / 2 c_{2}\right) q_{2}+\left(c_{1} / 2 c_{2}\right) q_{3}, p_{23}=\left(c_{2} / c_{1}\right) p_{13}+\left(1 / 2 c_{1}\right) q_{2}$, and $p_{33}=\left(1 / c_{1}\right) p_{13}+\left(1 / 2 c_{1} c_{2}\right) q_{2}+\left(1 / 2 c_{2}\right) q_{3}$. As a result, the 1-by-1, 2-by-2, and 3-by-3 leading principle minors of $P_{3}$ are, respectively, $\left.c_{1} p_{13}, c_{1} p_{13}\left(\left(c_{2}+1\right) / 2 c_{1}\right) q_{2}+\left(c_{1} / 2 c_{2}\right) q_{3}\right)$, and $(1 / 4) p_{13}\left[q_{2} q_{3}+\left(1 / c_{2}^{2}\right)\left(q_{2}+c_{1} q_{3}\right)^{2}\right]$. Hence $P_{3}$ is positive definite for any $p_{13}>0$. If we take $q_{2}=2 c_{2} p_{13}, q_{3}=$ $\left(4 c_{2} / c_{1}\right) p_{13}$ with $p_{13}>0$, then the condition (14) becomes $0<\kappa_{0}<\min \left\{r c_{1}^{2} c_{2} /\left(r^{2}+d^{2}\right)\left(2 c_{1}+c_{2}^{2}\right), 2 c_{1}^{2} c_{2} / 5 d\left(2 c_{1}+c_{2}^{2}\right)\right\}$. Thus when $c_{1}=24$ and $c_{2}=8$, the constraint on $\kappa_{0}$ can be calculated as $0<\kappa_{0}<576 / 35 \simeq 16.4571$. We choose $c_{1}=24$, $c_{2}=8$, and $\kappa_{0}=5$ for the simulation.

With the parameters chosen as above and by solving the optimization problem in (15), we can obtain that the maximum allowable upper bound of time-delays is $\tau_{0}=$ 1.2305. Assume that $\tau_{21}(t) \leq 0.4, \tau_{32}(t) \leq 0.6, \tau_{43}(t) \leq 0.9$, $\tau_{54}(t) \leq 0.7, \tau_{65}(t) \leq 0.5$, and $\tau_{16}(t) \leq 0.8$, all of which are piecewise constant functions of time $t$. The states of agents in the system (7) asymptotically reach consensus as shown in Figure 1.

\section{Conclusions}

This paper has studied the consensus problem for high-order MAS with directed network and asymmetric time-varying 
communication delays. An equivalent condition for the consensus convergence has been established based on an orthogonal linear transformation. A Lyapunov-like protocol design has been given, which is robust with respect to asymmetric time-varying communication delays and directed interactions. The maximum allowable upper bounds of delays have been determined by solving some optimization problems. Finally, numerical examples have been worked out to demonstrate the effectiveness of the theoretical results.

\section{Appendix}

\section{Proofs of Lemmas}

Proof of Lemma 3. From the property of $U_{c}$, let $U_{c}=$ $\left[(1 / \sqrt{N}) \mathbf{1}_{N} \quad v_{2} \quad \cdots \quad v_{N}\right]$ and $\widehat{U}=\left[\begin{array}{lll}v_{2} & \cdots & v_{N}\end{array}\right]$. Then $\mathscr{L} \mathbf{1}_{N}=0$ implies that

$$
\begin{aligned}
U_{c}^{T} \mathscr{L} U_{c} & =\left[\begin{array}{c}
\frac{1}{\sqrt{N}} \mathbf{1}_{N}^{T} \\
v_{2}^{T} \\
\vdots \\
v_{N}^{T}
\end{array}\right]\left[\begin{array}{llll}
0 & \mathscr{L} v_{2} & \cdots & \mathscr{L} v_{N}
\end{array}\right] \\
& =\left[\begin{array}{cccc}
0 & \frac{1}{\sqrt{N}} \mathbf{1}_{N}^{T} \mathscr{L} \nu_{2} & \cdots & \frac{1}{\sqrt{N}} \mathbf{1}_{N}^{T} \mathscr{L} v_{N} \\
0 & v_{2}^{T} \mathscr{L} v_{2} & \cdots & v_{2}^{T} \mathscr{L} v_{N} \\
\vdots & \vdots & \cdots & \vdots \\
0 & v_{N}^{T} \mathscr{L} \nu_{2} & \cdots & v_{N}^{T} \mathscr{L} v_{N}
\end{array}\right] .
\end{aligned}
$$

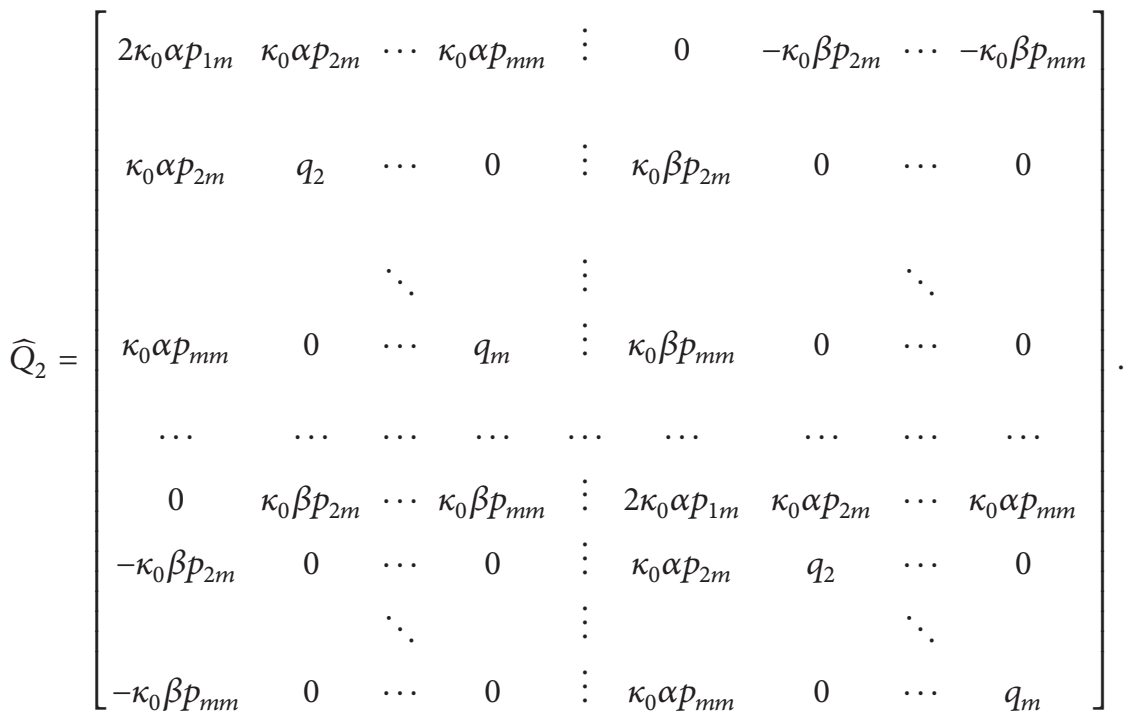

Denote the $k$-by- $k$ leading principle minor of $\widehat{Q}_{2}$ by $\widehat{Q}_{2}(k)$. For $\widehat{Q}_{2}(1), \ldots, \widehat{Q}_{2}(m)$, it follows by expanding them with respect to their last rows, respectively, that

$$
\widehat{Q}_{2}(1)=\kappa_{0} \alpha\left(2 p_{1 m}\right)
$$

Denote that $l=\left[(1 / \sqrt{N}) \mathbf{1}_{N}^{T} \mathscr{L} v_{2} \cdots(1 / \sqrt{N}) \mathbf{1}_{N}^{T} \mathscr{L} v_{N}\right]^{T}$ and $\widehat{L}=\widehat{U}^{T} \mathscr{L} \widehat{U}$. Then $U_{c}^{T} \mathscr{L} U_{c}$ is in the form of $\left[\begin{array}{ll}0 & l^{T} \\ 0 & \widehat{L}\end{array}\right]$. Due to the fact that $U_{c}^{T}=U_{c}^{-1}$ and similarity transformation does not change the eigenvalues of matrix, $\sigma(\mathscr{L})=\{0\} \cup \sigma(\widehat{L})$. It follows that $\sigma(\widehat{L}) \subset \sigma(\mathscr{L})$. If $\mathscr{G}$ is balanced, then $\mathbf{1}_{N}^{T} \mathscr{L}=0$. Thus $l=\left[\begin{array}{llll}(1 / \sqrt{N}) & \mathbf{1}_{N}^{T} \mathscr{L} \nu_{2} & \cdots & (1 / \sqrt{N}) \mathbf{1}_{N}^{T} \mathscr{L} \nu_{N}\end{array}\right]^{T}=0$. If $\mathscr{G}$ is strongly connected and balanced, according to Theorem 7 in [4], we obtain that $\left(\mathscr{L}^{T}+\mathscr{L}\right) / 2$ is a valid Laplacian matrix of an undirected graph. Then the strong connectivity of $\mathscr{G}$ implies that the corresponding graph of $\left(\mathscr{L}^{T}+\mathscr{L}\right) / 2$ is connected. Hence $\left(\mathscr{L}^{T}+\mathscr{L}\right) / 2$ is positive semidefinite and its rank is $N-1$. This proves that $\widehat{L}^{T}+\widehat{L}$ is positive definite.

Proof of Lemma 10. Denote $x=\left[\begin{array}{lll}x_{1} & \cdots & x_{m}\end{array}\right]^{T} \in \mathbb{R}^{m}$. Since $x^{T} P_{m} E_{m} x$ is a scalar, we can write $-x^{T} \widehat{Q}_{1} x=-\left(q_{2} x_{2}^{2}+\cdots+\right.$ $\left.q_{m} x_{m}^{2}\right)=x^{T}\left(E_{m}^{T} P_{m}+P_{m} E_{m}\right) x=2 x^{T} P_{m} E_{m} x=2\left[x_{2}\left(p_{11} x_{1}+\right.\right.$ $\left.\cdots+p_{m 1} x_{m}\right)+\cdots+x_{m}\left(p_{1(m-1)} x_{1}+\cdots+p_{m(m-1)} x_{m}\right)+$ $\left.\left(-c_{1} x_{2}-c_{2} x_{3}-\cdots-c_{m-1} x_{m}\right)\left(p_{1 m} x_{1}+\cdots+p_{m m} x_{m}\right)\right]$. The coefficient of the term $x_{1} x_{2}$ in $2 x^{T} P_{m} E_{m} x$ is $2\left(p_{11}-c_{1} p_{1 m}\right)$. From the equality of the left-hand and right-hand coefficients in $-x^{T} \widehat{Q}_{1} x=x^{T}\left(E_{m}^{T} P_{m}+P_{m} E_{m}\right) x$, we have $p_{11}=c_{1} p_{1 m}$. Notice that $p_{11}>0$ due to $P_{m}$ being positive definite. Then $c_{1}>0$ implies that $p_{1 m}>0$.

Let $\widehat{Q}_{2}=-\left(I_{2} \otimes E_{m}-\kappa_{0} D_{z} \otimes F_{m}\right)^{T}\left(I_{2} \otimes P_{m}\right)-\left(I_{2} \otimes P_{m}\right)\left(I_{2} \otimes\right.$ $\left.E_{m}-\kappa_{0} D_{z} \otimes F_{m}\right)$. In what follows, we only need to prove that $\widehat{Q}_{2}$ is positive definite when $\kappa_{0}$ satisfies (13). By directly computing, we obtain

$$
\widehat{Q}_{2}(k)=\kappa_{0} \alpha\left(\prod_{i=2}^{k} q_{i}\right)\left[2 p_{1 m}-\kappa_{0} \alpha\left(\sum_{i=2}^{k} \frac{p_{i m}^{2}}{q_{i}}\right)\right]
$$

$$
k=2, \ldots, m \text {. }
$$


For $\widehat{Q}_{2}(m+k), k=1, \ldots, m$, we first multiply the first row and the first column by $-\beta / \alpha$ and add to the $(m+1)$ th row and the $(m+1)$ th column, respectively. Then multiply, respectively, the second, third,...,mth, and $(m+1)$ th column by $-\kappa_{0} \alpha p_{2 m} / q_{2},-\kappa_{0} \alpha p_{3 m} / q_{3}, \ldots,-\kappa_{0} \alpha p_{m m} / q_{m}$, and $\alpha \beta /\left(\alpha^{2}+\right.$ $\left.\beta^{2}\right)$; add all of them to the first column. At last, expanding the resultant determinant with respect to the first column and making use of the results of $\widehat{Q}_{2}(k), k=1, \ldots, m$, we have

$$
\begin{aligned}
& \widehat{Q}_{2}(m+1) \\
& =2 \kappa_{0}^{2} \alpha p_{1 m} \prod_{i=2}^{m} q_{i}\left[2 \alpha p_{1 m}-\kappa_{0}\left(\alpha^{2}+\beta^{2}\right)\left(\sum_{i=2}^{m} \frac{p_{i m}^{2}}{q_{i}}\right)\right], \\
& \widehat{Q}_{2}(m+k) \\
& =\kappa_{0}^{2} \prod_{i=2}^{m} q_{i}\left[2 \alpha p_{1 m}-\kappa_{0}\left(\alpha^{2}+\beta^{2}\right)\left(\sum_{i=2}^{m} \frac{p_{i m}^{2}}{q_{i}}\right)\right] \\
& \quad \times \prod_{i=2}^{k} q_{i}\left[2 \alpha p_{1 m}-\kappa_{0}\left(\alpha^{2}+\beta^{2}\right)\left(\sum_{i=2}^{k} \frac{p_{i m}^{2}}{q_{i}}\right)\right], \\
& k=2, \ldots, m .
\end{aligned}
$$

Hence $\widehat{Q}_{2}$ is positive definite if and only if $\widehat{Q}_{2}(k)>0$ for all $k=1, \ldots, 2 m$. From the assumption, it is easy to see that $\widehat{Q}_{2}(1)>0$. Since $P_{m}$ is positive definite, $p_{m m}>0$. As a result, $\sum_{i=2}^{m}\left(p_{i m}^{2} / q_{i}\right)>0$. In addition, it is not hard to get $\sum_{i=2}^{k}\left(p_{i m}^{2} / q_{i}\right)=0$ if and only if $p_{2 m}=\cdots=p_{k m}=0$. Consider $\widehat{Q}_{2}(2), \ldots, \widehat{Q}_{2}(m)$. Suppose that $k^{\prime} \in\{2, \ldots, m-1\}$ which satisfies $p_{2 m}=\cdots=p_{k^{\prime} m}=0$ and $p_{\left(k^{\prime}+1\right) m} \neq 0$. Then we have $\sum_{i=2}^{k}\left(p_{i m}^{2} / q_{i}\right)=0$ for $k=2, \ldots, k^{\prime}$ and $\sum_{i=2}^{k}\left(p_{i m}^{2} / q_{i}\right)>0$ for $k=k^{\prime}+1, \ldots, m$. Consequently, $\widehat{Q}_{2}(2)>0, \ldots, \widehat{Q}_{2}\left(k^{\prime}\right)>0$ for any $\kappa_{0}>0$, and $\widehat{Q}_{2}\left(k^{\prime}+1\right)>0, \ldots, \widehat{Q}_{2}(m)>0$ if and only if $\kappa_{0}<2 p_{1 m} / \alpha \sum_{i=2}^{m}\left(p_{i m}^{2} / q_{i}\right)$. Next, consider $\widehat{Q}_{2}(m+$ $1), \ldots, \widehat{Q}_{2}(2 m)$. It is evident that $\widehat{Q}_{2}(m+1)>0$ if and only if $\kappa_{0}$ satisfies (13). Following the similar guidelines of the proof of $\widehat{Q}_{2}(2), \ldots, \widehat{Q}_{2}(m)$, we have $\widehat{Q}_{2}(m+2)>0, \ldots, \widehat{Q}_{2}(2 m)>0$ if and only if $\kappa_{0}$ satisfies (13). By summing up the discussion above and making use of the fact that $\alpha /\left(\alpha^{2}+\beta^{2}\right) \leq 1 / \alpha$, we obtain the first conclusion. The second conclusion follows directly from the first one.

To prove Lemma 11, we introduce the following result.

Lemma A.1 (see Theorem 4.6 in [20]). Consider $E_{m}$ and $F_{m}$ in (5). Suppose graph $\mathscr{G}(\mathscr{A})$ contains a spanning tree; the associated Laplacian matrix is $\mathscr{L}, U_{c}^{T} \mathscr{L} U_{c}=\left[\begin{array}{cc}0 & l^{T} \\ 0 & \widehat{L}\end{array}\right]\left(U_{c}\right.$ is given as in Lemma 3); the parameters $c_{i}, i \in \underline{m-1}$, are chosen such that $E_{m}$ has only one zero eigenvalue and other nonzero
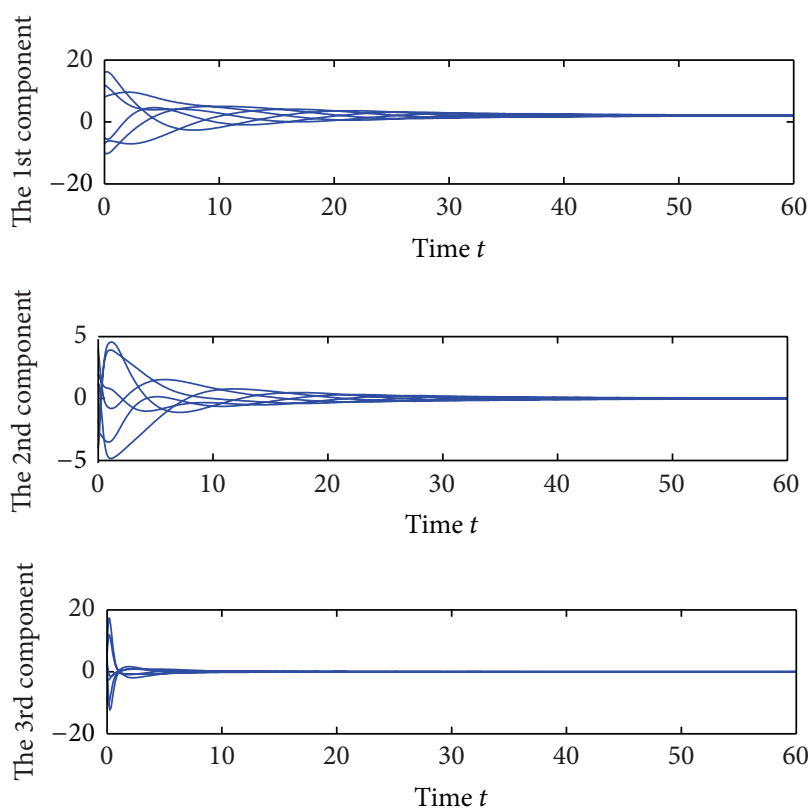

Figure 1: Trajectories of the first, the second, and the third state components of six agents with a directed circle and asymmetric time-varying delays.

eigenvalues have negative real parts. If there exist some positive definite matrices $P, Q$ and a positive real number $\kappa_{0}$ such that

$$
\begin{aligned}
& {\left[I_{2} \otimes E_{m}-\kappa_{0}\left(D_{1} \otimes F_{m}\right)\right]^{T} P} \\
& +P\left[I_{2} \otimes E_{m}-\kappa_{0}\left(D_{1} \otimes F_{m}\right)\right]<0, \\
& {\left[I_{2} \otimes E_{m}-\kappa_{0}\left(D_{2} \otimes F_{m}\right)\right]^{T} Q} \\
& +Q\left[I_{2} \otimes E_{m}-\kappa_{0}\left(D_{2} \otimes F_{m}\right)\right]<0,
\end{aligned}
$$

then $I_{N-1} \otimes E_{m}-\kappa_{0} \widehat{L} \otimes F_{m}$ is Hurwitz stable, where $D_{1}=$ $\left[\begin{array}{cc}r & d \\ -d & r\end{array}\right], D_{2}=\left[\begin{array}{cc}2 d & d \\ -d & 2 d\end{array}\right], r=\min \{\operatorname{Re}(\lambda): 0 \neq \lambda \in \sigma(\mathscr{L})\}$, $d=\max _{i \in \underline{N}}\left\{d_{i}\right\}$ is the maximum vertex in-degree over $\mathscr{G}(\mathscr{A})$.

Proof of Lemma 11. We only need to prove that the inequalities in (A.5) hold. Take $P=Q=I_{2} \otimes P_{m}$. From Lemma 10, (14) implies (A.5). The proof is completed.

\section{Conflict of Interests}

The author declares that there is no conflict of interests regarding the publication of this paper.

\section{Acknowledgments}

This work was supported by the National Natural Science Foundation of China under Grant no. 61304163, the Natural Science Foundation of Shandong Province (no. ZR2013FQ008), and the Independent Innovation Foundation of Shandong University (no. 2013ZRQP006). 


\section{References}

[1] V. Borkar and P. P. Varaiya, "Asymptotic agreement in distributed estimation," IEEE Transactions on Automatic Control, vol. 27, no. 3, pp. 650-655, 1982.

[2] J. N. Tsitsiklis and M. Athans, "Convergence and asymptotic agreement in distributed decision problems," IEEE Transactions on Automatic Control, vol. 29, no. 1, pp. 42-50, 1984.

[3] A. Jadbabaie, J. Lin, and A. S. Morse, "Coordination of groups of mobile autonomous agents using nearest neighbor rules," IEEE Transactions on Automatic Control, vol. 48, no. 6, pp. 988-1001, 2003.

[4] R. Olfati-Saber and R. M. Murray, "Consensus problems in networks of agents with switching topology and time-delays," IEEE Transactions on Automatic Control, vol. 49, no. 9, pp. 15201533, 2004.

[5] W. Ren and R. W. Beard, "Consensus seeking in multiagent systems under dynamically changing interaction topologies," IEEE Transactions on Automatic Control, vol. 50, no. 5, pp. 655661, 2005.

[6] G. Xie and L. Wang, "Consensus control for a class of networks of dynamic agents," International Journal of Robust and Nonlinear Control, vol. 17, no. 10-11, pp. 941-959, 2007.

[7] B. Liu, G. Xie, Y. Gao, J. Wu, J. Zhang, and W. Luo, "Consensus analysis of second-order multiagent systems with general topology and time delay," Journal of Applied Mathematics, vol. 2013, Article ID 359750, 8 pages, 2013.

[8] W. Ren, K. Moore, and Y. Chen, "High-order and model reference Luo, Wenguangconsensus algorithms in cooperative control of multi-vehicle systems," Journal of Dynamic Systems, Measurement, and Control, vol. 129, no. 5, pp. 678-688, 2007.

[9] P. Wieland, J. Kim, H. Scheu, and F. Allgower, "On consensus in multi-agent systems with linear high-order agents," in Proceedings of the 17th World Congress, International Federation of Automatic Control (IFAC '08), pp. 1541-1546, Seoul, Korea, July 2008.

[10] W. Yu, G. Chen, W. Ren, J. Kurths, and W. X. Zheng, "Distributed higher order consensus protocols in multiagent dynamical systems," IEEE Transactions on Circuits and Systems I: Regular Papers, vol. 58, no. 8, pp. 1924-1932, 2011.

[11] D. Lee and M. W. Spong, "Agreement with non-uniform information delays," in Proceedings of the American Control Conference, pp. 756-761, Minneapolis, Minn, USA, June 2006.

[12] J. Qin, H. Gao, and W. X. Zheng, "A new result on average consensus for multiple agents with switching topology and communication delay," in Proceedings of the 48th IEEE Conference on Decision and Control held jointly with 28th Chinese Control Conference (CDC/CCC '09), pp. 3703-3708, Shanghai, China, December 2009.

[13] P. Lin and Y. Jia, "Consensus of a class of second-order multiagent systems with time-delay and jointly-connected topologies," IEEE Transactions on Automatic Control, vol. 55, no. 3, pp. 778-784, 2010.

[14] P. Bliman and G. Ferrari-Trecate, "Average consensus problems in networks of agents with delayed communications," Automatica, vol. 44, no. 8, pp. 1985-1995, 2008.

[15] Y. G. Sun and L. Wang, "Consensus problems in networks of agents with double-integrator dynamics and time-varying delays," International Journal of Control, vol. 82, no. 10, pp. 19371945, 2009.

[16] Y. G. Sun and L. Wang, "Consensus of multi-agent systems in directed networks with uniform time-varying delays," IEEE
Transactions on Automatic Control, vol. 54, no. 7, pp. 1607-1613, 2009.

[17] U. Münz, A. Papachristodoulou, and F. Allgöwer, "Consensus reaching in multi-agent packet-switched networks with nonlinear coupling," International Journal of Control, vol. 82, no. 5, pp. 953-969, 2009.

[18] C. Godsil and G. Royle, Algebraic Graph Theory, vol. 207 of Graduate Texts in Mathematics, Springer, New York, NY, USA, 2001.

[19] Y. G. Sun, L. Wang, and G. Xie, "Average consensus in networks of dynamic agents with switching topologies and multiple timevarying delays," Systems \& Control Letters, vol. 57, no. 2, pp. 175$183,2008$.

[20] F. Jiang and L. Wang, "Consensus seeking of high-order dynamic multi-agent systems with fixed and switching topologies," International Journal of Control, vol. 83, no. 2, pp. 404420, 2010.

[21] K. Gu, V. L. Kharitonov, and J. Chen, Stability of Time-Delay Systems, Birkhäuser, Boston, Mass, USA, 2003.

[22] B. Boyd, L. El Ghaoui, E. Feron, and V. Balakrishnan, Linear Matrix Inequalities in System and Control Theory, SIAM, Philadelphia, Pa, USA, 1994. 


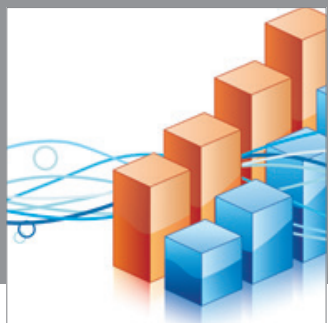

Advances in

Operations Research

mansans

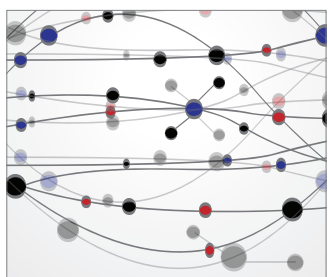

The Scientific World Journal
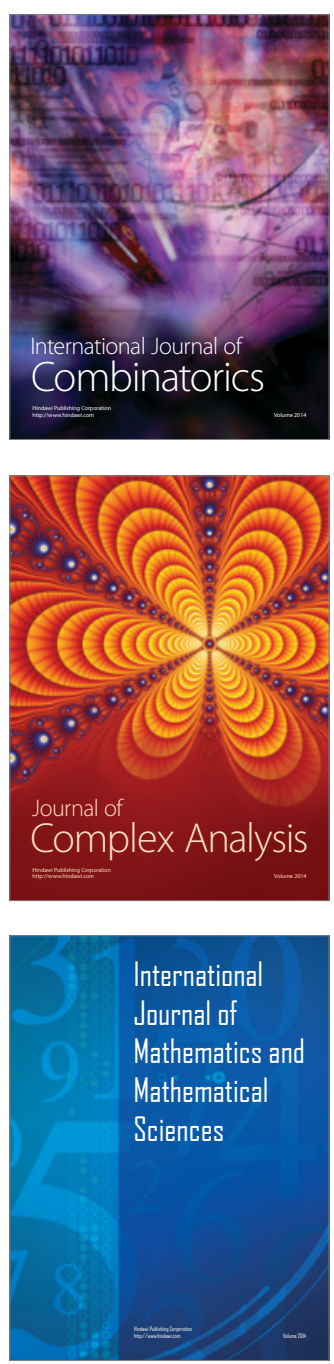
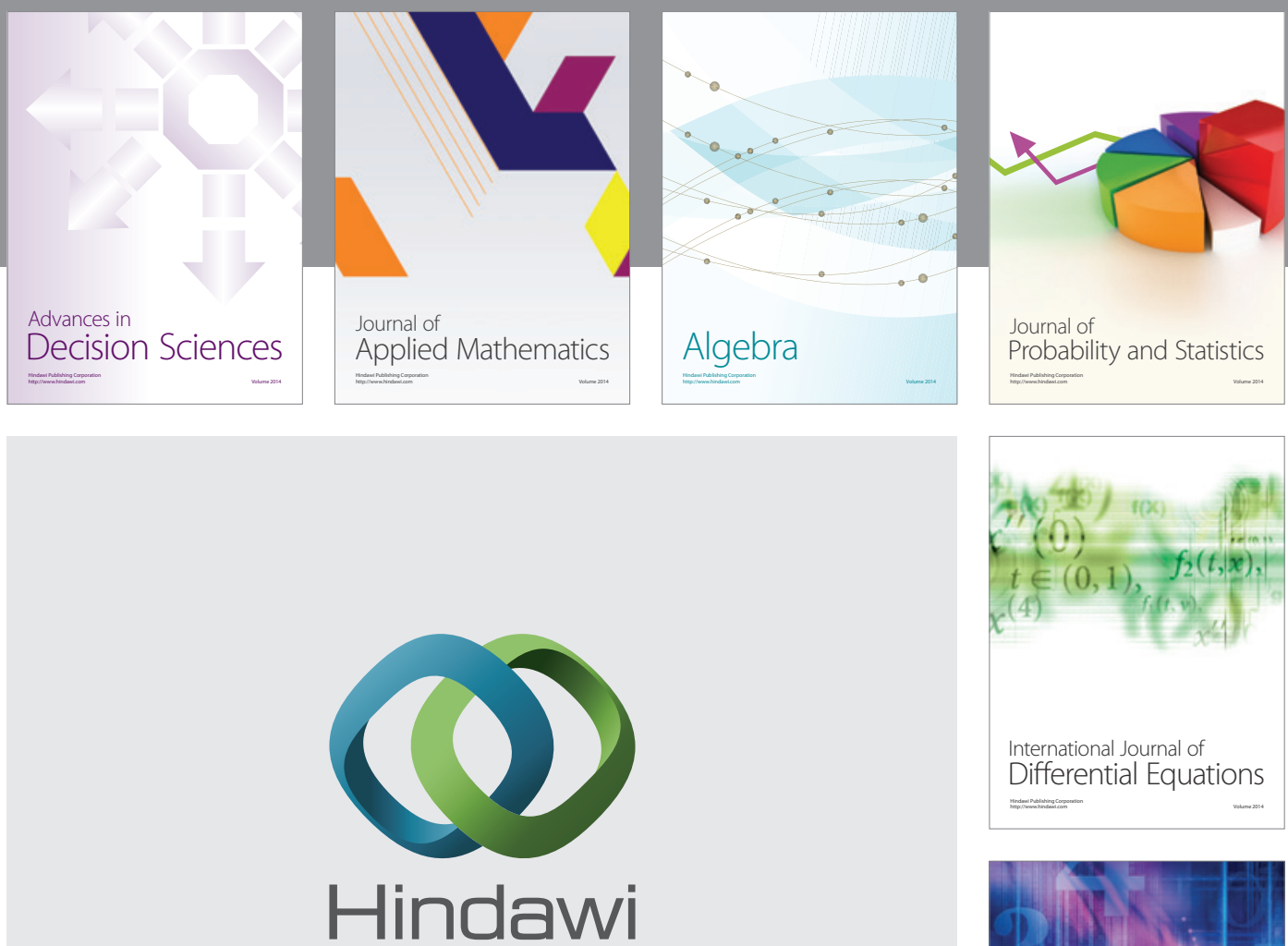

Submit your manuscripts at http://www.hindawi.com
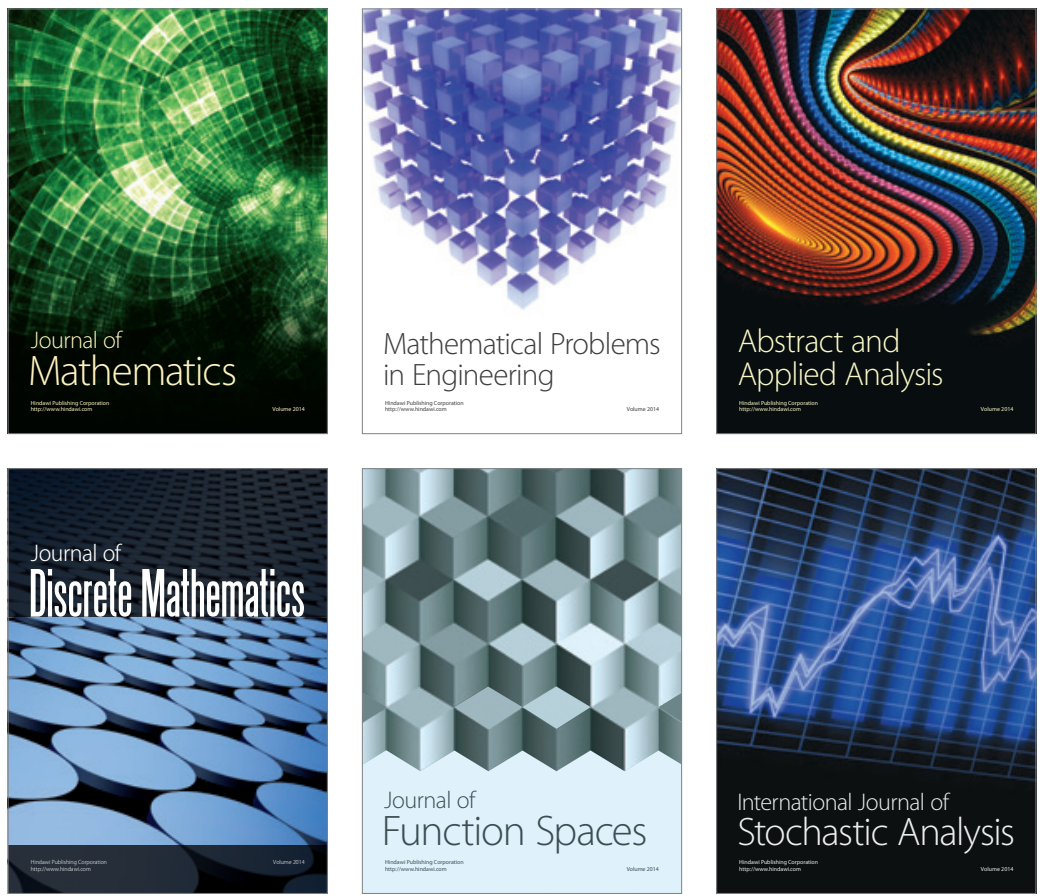

Journal of

Function Spaces

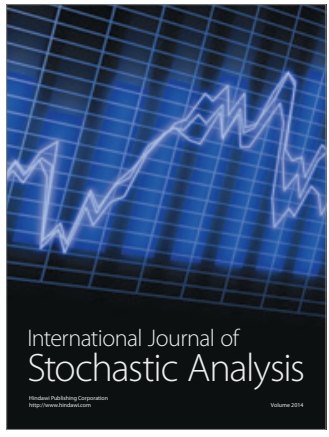

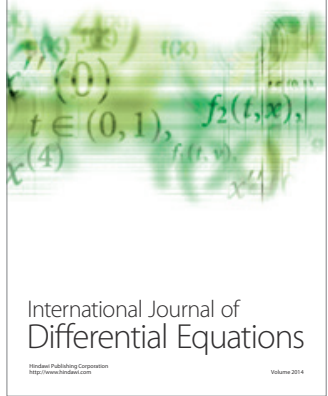
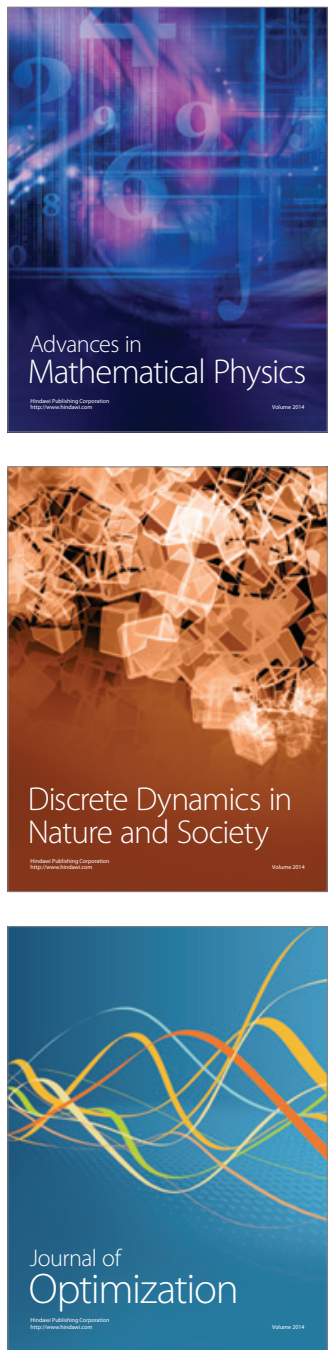